\title{
Computability via the Lambda Calculus with Patterns
}

\author{
Bodin Skulkiat (Corresponding author) \\ Department of Mathematics, Faculty of Science \\ Chulalongkorn University, Bangkok, Thailand \\ Tel: 66-89-666-5804 E-mail: skulkiat.b@gmail.com \\ Pimpen Vejjajiva \\ Department of Mathematics, Faculty of Science \\ Chulalongkorn University, Bangkok, Thailand \\ E-mail: pimpen@abhisit.org \\ Mark E. Hall \\ Department of Mathematics and Computer Science, Hastings College \\ 710 North Turner Ave, Hastings, NE 68901, USA \\ E-mail: mhall@hastings.edu
}

\begin{abstract}
We introduce a concept of computability relative to a structure, which specifies which functions on the universe of a first-order structure are computable, using the lambda calculus with patterns. In doing so, we add a new congruence, $\equiv_{\mathfrak{A}}$, called congruence in a structure to identify two syntactically different terms which represent the same element of the universe. We then show that, with the introduction of the new congruence, all the basic properties of the original lambda calculus with patterns still hold, including the Church-Rosser theorem.
\end{abstract}

Keywords: Computability, Lambda calculus, Lambda calculus with patterns, Congruence in a structure

\section{Introduction}

Most theories of computability are still limited to functions on the natural numbers, or generalizations thereof, e.g., Santos 1971, Blum, Shub, \& Smale 1989, and Koepke 2005. However, there is no obvious reason why we cannot consider computability in a very general setting, since computability is really just talking about whether we can produce an answer by carrying out a finite number of precisely specified elementary processing steps. There are two basic questions that need to be answered in order to do this: Which processing steps are elementary and how do we specify what steps to carry out in order to perform a particular computation?

In this paper, we extend the concept of computability to functions on the domain of an arbitrary first-order structure by taking a software-oriented approach: A function is computable if and only if we can write a "program" to compute it, assuming that the computation of every function and relation in the structure is an elementary processing step. A good, mathematically rigorous "programming language" is the lambda calculus. Since its introduction by Alonzo Church in the 1930's the lambda calculus has been studied in many aspects, notably regarding computability. A history of lambda calculus related to computation theory can be found in Barendregt, 1997 and Cardone \& Hindley, 2006. For an introduction to the original lambda calculus see Hindley \& Seldin, 1986, and for a more in-depth analysis see Barendregt, 2001. Since the lambda calculus only deals with symbols, without any assumptions about their meanings, it is a good tool to help us extend the concept of computability to functions on the domain of an arbitrary first-order structure. To gain greater expressive power, we will use a lambda calculus with patterns (Vejjajiva, 1997; Vejjajiva \& Hall, 2002), which we will briefly describe.

Assume there are given an infinite sequence of distinct symbols, called variables, and a set of symbols which are distinct from the variables, called constants. The set of patterns is defined inductively as follows.

$\mathrm{P} 1$. Each variable and constant is a pattern.

P2. If $P_{1}$ is a pattern which is not a variable, $P_{2}$ is any pattern, and no variable occurs in both $P_{1}$ and $P_{2}$, then $\left(P_{1} P_{2}\right)$ is a pattern.

Then, the set of terms is defined inductively as follows.

T1. Each variable and constant is a term, called an atom.

T2. If $M$ and $N$ are any terms, $(M N)$ is a term, called an application.

T3. If $P$ is any pattern and $Q$ is any term, $(\lambda P . Q)$ is a term, called a simple abstraction. 
T4. If $P$ is any pattern, $Q$ is any term, and $A$ is any abstraction, $((\lambda P . Q) \mid A)$ is a term, called a compound abstraction.

An abstraction $(\lambda x . M)$ represents a function $f: x \mapsto M$. For example, $(\lambda x . x)$ represents an identity function. An application $(M N)$ represents applying a function represented by $M$ to an argument represented by $N$. For example, if we let $\mathbf{0}$ be a constant representing the natural number $0,((\lambda x . x) 0)$ represents applying an identity function to 0 , which would result in 0 . Avoiding complex technical details for the moment, we will use the symbol $\triangleright$ to represent the idea of "computing". In this notation the preceding example can be written as $((\lambda x \cdot x) \mathbf{0}) \triangleright \mathbf{0}$. Here is a more involved example. If we let $\mathbf{S}$ be a constant representing the successor function and $\bar{a}$ be a constant representing any natural number $a$, then $((\lambda \mathbf{0} . \mathbf{0}) \mid(\lambda \mathbf{S} x . x))$ represents a predecessor function which maps $0 \mapsto 0$, i.e. $(((\lambda \mathbf{0 . 0}) \mid(\lambda \mathbf{S} x . x)) \mathbf{0}) \triangleright \mathbf{0}$, and maps $(a+1) \mapsto a$, i.e. $(((\lambda \mathbf{0 . 0}) \mid(\lambda \mathbf{S} x . x)) \mathbf{S} \bar{a}) \triangleright \bar{a}$.

The general idea of how to extend the concept of computable functions to a first-order structure $\mathfrak{A}$ for a language $\mathcal{L}$ is as follows. For each element $a \in|\mathfrak{A}|$, let $\bar{a}$ be a distinct symbol that does not occur in $\mathcal{L}$. Define patterns and terms as in the lambda calculus with patterns, using as constants all of the symbols in $\mathcal{L}$ together with all of the symbols $\bar{a}$. Then an $n$-ary function $g$ on $|\mathfrak{A}|$ is computable relative to $\mathfrak{A}$ if and only if there is a term $G$ such that for all $a_{1}, \ldots, a_{n}, a \in|\mathfrak{A}|$ we have $G \bar{a}_{1} \ldots \bar{a}_{n} \triangleright \bar{a}$, whenever $g\left(a_{1}, \ldots, a_{n}\right)=a$. Informally speaking, a function on $|\mathfrak{A}|$ is computable relative to $\mathfrak{A}$ if and only if it can be represented by a term which captures all its functionalities. The interpretations of the elements of $\mathcal{L}$ in the structure $\mathfrak{A}$ are captured by adding a new congruence, $\equiv_{\mathfrak{A}}$, called congruence in a structure, to identify two syntactically different terms that represent the same element of the domain $|\mathfrak{A}|$. For example, $\mathbf{S} \overline{0} \equiv_{\mathfrak{A}} \overline{1}$, since they both represent 1 in $\mathbb{N}$. We then show that, with the introduction of the new congruence, all the basic properties of the original lambda calculus with patterns still hold, particularly the Church-Rosser theorem.

\section{$2 \lambda$ P-terms and Preliminary Lemmas}

\section{$2.1 \lambda$-terms}

Definition 2.1.1 is based on the lambda calculus with patterns (Vejjajiva, 1997; Vejjajiva \& Hall, 2002) with some adjustments. Let $\mathcal{L}$ be a first-order language and $\mathfrak{A}$ a structure for $\mathcal{L}$. We use $|\mathfrak{A}|$ to denote the domain of $\mathfrak{A}$.

Definition 2.1.1. For each element $a$ in $|\mathfrak{A}|$, let $\bar{a}$ be a distinct symbol that is not in $\mathcal{L}$. We call all the nonlogical symbols in $\mathcal{L}$ together with all of the symbols $\bar{a}$ and two additional distinct symbols T and $\mathrm{F}$ constants. Assume also that an infinite sequence of distinct symbols $v_{1}, v_{2}, \ldots$, called variables, is given. Patterns and $\lambda \mathbf{P}$-terms are expressions constructed using these symbols, as follows.

The set of patterns is the smallest set of expressions satisfying the following.

P1. All variables are patterns.

P2. The two constant symbols $\mathrm{T}$ and $\mathrm{F}$, and all constant symbols in $\mathcal{L}$ are patterns.

P3. All function symbols $f$ in $\mathcal{L}$ such that $f^{\mathfrak{A}}$ is one-to-one are patterns.

P4. If $P$ is a pattern that is not a variable, $Q$ is any pattern, and no variable occurs in both $P$ and $Q$, then $(P Q)$ is a pattern.

The set of $\lambda \mathbf{P}$-terms is divided into sets of atoms, applications, and abstractions, and is defined to be the smallest set of expressions satisfying the following.

T1. All variables and constants are $\lambda$ P-terms (these are the atoms).

T2. If $P$ and $Q$ are any $\lambda \mathrm{P}$-terms, then $(P Q)$ is a $\lambda \mathrm{P}$-term (these are the applications).

T3. If $P$ is any pattern and $Q$ is any $\lambda \mathrm{P}$-term, then $(\lambda P . Q)$ is a $\lambda \mathrm{P}$-term (called a simple abstraction).

T4. If $P$ is any pattern, $Q$ is any $\lambda \mathrm{P}$-term, and $A$ is any abstraction, then $((\lambda P . Q) \mid A)$ is a $\lambda \mathrm{P}$-term (called a compound abstraction).

An abstraction is either a simple abstraction or a compound abstraction.

\section{Notation.}

i. Parentheses will be omitted by using the convention of association to the left.

ii. $\lambda P . M N$ will abbreviate $(\lambda P .(M N))$.

iii. We may simply write "terms" for " $\lambda$ P-terms".

iv. Syntactic identity of terms will be denoted by $\equiv$.

v. The set of free variables of a term $M$ will be denoted by $F V(M)$.

vi. If $\underline{x}=x_{1}, \ldots, x_{k}$ is a sequence of distinct variables, $\underline{N}=N_{1}, \ldots, N_{k}$ is a sequence of terms, and $M$ is a term, then the result of simultaneously substituting $N_{1}, \ldots, \overline{N_{k}}$ for all free occurrences of $x_{1}, \ldots, x_{k}$, respectively, in $M$ (subject to avoiding clashes of variables) is denoted by $[\underline{N} / \underline{x}] M$.

vii. We will say that two terms are of the same form whenever they are both atoms, both applications, both simple abstractions, or both compound abstractions.

The definitions of bound and free occurrences of a variable, simultaneous substitution, and a change of bound variable $(\alpha$-step) are analogous to those in the original lambda calculus. 


\subsection{Preliminary Lemmas from Previous Work}

The following lemmas and notes are from (Vejjajiva, 1997).

Lemma 2.2.1. Let $\underline{x}=x_{1}, \ldots, x_{k}, k \geq 1$, be distinct variables, $M, \underline{N}=N_{1}, \ldots, N_{k}$ be terms, and $\lambda P . Q$ be a simple abstraction.

a. If $\left\{x_{1}, \ldots, x_{k}\right\} \cap F V(M)=\left\{x_{i_{1}}, \ldots, x_{i_{m}}\right\}$, then $\left[N_{1} / x_{1}, \ldots, N_{k} / x_{k}\right] M \equiv\left[N_{i_{1}} / x_{i_{1}}, \ldots, N_{i_{m}} / x_{i_{m}}\right] M$.

b. If $F V(P) \cap F V\left(x_{1} \ldots x_{k} N_{1} \ldots N_{k}\right)=\emptyset$, then $[\underline{N} / \underline{x}](\lambda P . Q) \equiv \lambda P .[\underline{N} / \underline{x}] Q$.

Lemma 2.2.2. Let $M$ and $N$ be terms such that $M \equiv_{\alpha} N$.

a. If $M \equiv M_{1} M_{2}$, then $N \equiv N_{1} N_{2}$ for some terms $N_{1}$ and $N_{2}$, where $M_{i} \equiv_{\alpha} N_{i}, i=1,2$;

$b$. if $M \equiv \lambda P . Q$, and no variable in $P$ has been changed, then $N \equiv \lambda P \cdot Q^{\prime}$ for some term $Q^{\prime}$ such that $Q \equiv{ }_{\alpha} Q^{\prime}$;

c. if $M \equiv(\lambda P . Q \mid A)$ then $N \equiv\left(\lambda P^{\prime} \cdot Q^{\prime} \mid A^{\prime}\right)$ for some abstractions $\lambda P^{\prime} \cdot Q^{\prime}$ and $A^{\prime}$ where $\lambda P \cdot Q \equiv_{\alpha} \lambda P^{\prime} \cdot Q^{\prime}$ and $A \equiv_{\alpha} A^{\prime}$.

\section{Lemma 2.2.3.}

a. For any terms $M$ and $N$, if $M \equiv_{\alpha} N$, then $F V(M)=F V(N)$.

$b$. For any term $M$, any variables $x_{1}, \ldots, x_{n}, n \geq 1$, there exists a term $M^{\prime}$ such that $M \equiv_{\alpha} M^{\prime}$ and none of $x_{1}, \ldots, x_{n}$ is bound in $M^{\prime}$.

Lemma 2.2.4. Let $\underline{x}=x_{1}, \ldots, x_{k}, k \geq 1$ be distinct variables, and $\underline{N}=N_{1}, \ldots, N_{k}, \underline{N}^{\prime}=N_{1}^{\prime}, \ldots, N_{k}^{\prime}$ be terms such that $N_{i} \equiv_{\alpha} N_{i}^{\prime}$ for all $1 \leq i \leq k$. For any terms $M$ and $M^{\prime}$, if $M \equiv_{\alpha} M^{\prime}$, then $[\underline{N} / \underline{x}] M \equiv_{\alpha}\left[\underline{N^{\prime}} / \underline{x}\right] M^{\prime}$.

\section{Computability Relative to a Structure}

\subsection{Congruence in a Structure}

Definition 3.1.1. Single-Step Congruence in $\mathfrak{A}$, denoted by $\equiv_{1 \mathfrak{A}}$, is defined as follows.

$\mathrm{C} 1$. For any constant symbol $c$ in $\mathcal{L}$ and any $a$ in $|\mathfrak{A}|, c \equiv_{1 \mathfrak{A}} \bar{a}$ if $c^{\mathfrak{A}}=a$.

C2. For any $n$-ary function symbol $f$ in $\mathcal{L}$ and any $a, a_{1}, \ldots, a_{n}$ in $|\mathfrak{A}|, f \bar{a}_{1} \ldots \bar{a}_{n} \equiv_{1 \mathfrak{A}} \bar{a}$ if $f^{\mathfrak{A}}\left(a_{1}, \ldots, a_{n}\right)=a$.

C3. For any $n$-ary relation symbol $r$ in $\mathcal{L}$ and any $a_{1}, \ldots, a_{n}$ in $|\mathfrak{A}|, r \bar{a}_{1} \ldots \bar{a}_{n} \equiv_{1 \mathfrak{A}} \begin{cases}\mathrm{T} & \text { if }\left(a_{1}, \ldots, a_{n}\right) \in r^{\mathfrak{A}}, \\ \mathrm{F} & \text { otherwise. }\end{cases}$

C4. For any terms $M$ and $N, M \equiv_{1 \mathfrak{A}} N$ if $N \equiv_{1 \mathfrak{A}} M$ by C1, C2, or C3.

C5. Let $P$ be any pattern; $A$ be any abstraction; and $M, N$, and $Q$ be any terms such that $M \equiv_{1 \mathfrak{A}} N$. Then
i. $M Q \equiv_{1 \mathfrak{A}} N Q$.
ii. $Q M \equiv_{1 \mathfrak{A}} Q N$.
iii. $\lambda P . M \equiv_{1 \mathfrak{A}} \lambda P . N$.
iv. $(\lambda P . M \mid A) \equiv_{1 \mathfrak{A}}(\lambda P . N \mid A)$.
v. $(\lambda P . Q \mid M) \equiv_{1 \mathfrak{A}}(\lambda P . Q \mid N)$ if $M$ and $N$ are abstractions.

For any terms $M$ and $N$, we write $M \equiv_{1 \mathfrak{A}}^{0} N$ if $M \equiv_{1 \mathfrak{A}} N$ by C1, C2, C3, or C4.

If $L$ is an occurrence of a term $M$ in a term $Q$ and $M \equiv_{1 \mathfrak{A}}^{0} N$, the act of replacing $L$ by $N$ is called a $1 \mathfrak{A}$-conversion in $Q$.

\section{Note 3.1.2.}

a. If $M \equiv_{1 \mathfrak{A}} N$ where $M$ and $N$ are terms which are not atomic, then $M \not_{1 \mathfrak{A}}^{0} N$.

b. If $M \equiv_{1 \mathfrak{A}} N$ and $F V(M) \cup F V(N) \neq \emptyset$, then $M \not_{1 \mathfrak{A}}^{0} N$.

c. If $M \equiv_{1 \mathfrak{A}} N$ but $M \not_{1 \mathfrak{A}}^{0} N$, then $M$ and $N$ are of the same form.

d. For any variable $x$ and any term $M, x \neq_{1 \mathfrak{A}} M$.

Definition 3.1.3. For any terms $M$ and $N$, we say $M$ is congruent in $\mathfrak{A}$ to $N$, denoted by $M \equiv_{\mathfrak{A}} N$, if there exists a sequence of terms $M \equiv M_{1}, \ldots, M_{n} \equiv N, n \geq 1$, such that for each $1 \leq i<n, M_{i} \equiv_{1 \mathfrak{A}} M_{i+1}$.

If $L$ is an occurrence of a term $M$ in a term $Q$ and $M \equiv_{\mathfrak{A}} N$, the act of replacing $L$ with $N$ is called an $\mathfrak{A}$-conversion in $Q$.

\section{Note 3.1.4.}

a. If $M \equiv_{\mathfrak{A}} N$ and $M$ contains an abstraction then $M$ and $N$ are of the same form.

b. If $M_{1} M_{2} \equiv_{\mathfrak{A}} N_{1} N_{2}$ with no $\equiv_{1 \mathfrak{A}}^{0}$ in the sequence of congruences, then $M_{1} \equiv_{\mathfrak{A}} N_{1}$ and $M_{2} \equiv_{\mathfrak{A}} N_{2}$.

Lemma 3.1.5. The relation $\equiv_{\mathfrak{A}}$ is an equivalence relation.

Proof. It is clear that $\equiv_{\mathfrak{A}}$ is reflexive and transitive. By induction, it can be shown that $\equiv_{\mathfrak{A}}$ is symmetric.

Lemma 3.1.6. For any $a, b$ in $|\mathfrak{A}|$, if $\bar{a} \equiv_{\mathfrak{A}} \bar{b}$ then $a=b$.

Proof. By inducting on the length of the sequence of congruences. 
Lemma 3.1.7. Let $P$ be a pattern with $F V(P)=\left\{x_{1}, \ldots, x_{k}\right\}, k \geq 1$, and $\underline{U}=U_{1}, \ldots, U_{k}, \underline{V}=V_{1}, \ldots$, $V_{k}$ be terms. Let $\underline{x}=x_{1}, \ldots, x_{k}$. If $[\underline{U} / \underline{x}] P \equiv_{\mathfrak{A}}[\underline{V} / \underline{x}] P$, then $U_{i} \equiv_{\mathfrak{A}} V_{i}$ for all $1 \leq i \leq k$.

Proof. Assume $[\underline{U} / \underline{x}] P \equiv_{\mathfrak{A}}[\underline{V} / \underline{x}] P$. Induct on $P$. The proof for the case $P \equiv x_{1}$ is easy. Assume $P \equiv P_{1} P_{2}$.

Let $[\underline{U} / \underline{x}] P \equiv_{\mathfrak{A}}[\underline{V} / \underline{x}] P$ by a sequence of terms $[\underline{U} / \underline{x}] P \equiv K_{1}, \ldots, K_{s} \equiv[\underline{V} / \underline{x}] P, s \geq 1$.

Case 1. $K_{i} \not_{1 \mathfrak{A}}^{0} K_{i+1}$ for all $1 \leq i<s$.

By Note 3.1.4(b) $[\underline{U} / \underline{x}] P_{1} \equiv_{\mathfrak{A}}[\underline{V} / \underline{x}] P_{1}$ and $[\underline{U} / \underline{x}] P_{2} \equiv_{\mathfrak{A}}[\underline{V} / \underline{x}] P_{2}$, so by induction, $U_{i} \equiv_{\mathfrak{A}} V_{i}$ for all $1 \leq i \leq k$.

Case 2. $K_{i} \equiv_{1 \mathfrak{A}}^{0} K_{i+1}$ for some $1 \leq i<s$.

Let $n$ be the first such $i$. Then $K_{n} \equiv f \bar{a}_{1} \ldots \bar{a}_{q}$ and $K_{n+1} \equiv \bar{a}$ for some function symbol $f$, and some $a, a_{1}, \ldots, a_{q} \in|\mathfrak{A}|, q \geq 1$. Since $K_{n+1} \equiv_{\mathfrak{A}} K_{s} \equiv[\underline{V} / \underline{x}] P$, by Note $3.1 .2 K_{j} \equiv_{1 \mathfrak{A}}^{0} K_{j+1}$ for some $n+1 \leq j<s$. Let $m$ be the most such $j$. Then we have $K_{m} \equiv \bar{b}$ and $K_{m+1} \equiv g \bar{b}_{1} \ldots \bar{b}_{r}$ for some function symbol $g$, and some $b, b_{1}, \ldots, b_{r} \in|\mathfrak{A}|, r \geq 1$. Since a pattern cannot begin with a variable and $[\underline{U} / \underline{x}] P \equiv_{\mathfrak{A}} K_{n} \equiv f \bar{a}_{1} \ldots \bar{a}_{q}$ with $K_{j} \not_{1 \mathfrak{A}}^{0} K_{j+1}$ for all $1 \leq j<n$, by induction on $q$, the pattern $P$ must begin with $f$. Similarly for $g \bar{b}_{1} \ldots \bar{b}_{r} \equiv K_{m+1} \equiv_{\mathfrak{A}}[\underline{V} / \underline{x}] P$, the pattern $P$ must begin with $g$. Therefore $f \equiv g$. By Lemma 3.1 .6 and the fact that $f^{\mathfrak{A}}$ is one-to-one, we have $q=r$ and $a_{j}=b_{j}$ for all $1 \leq j \leq q$. Then $[\underline{U} / \underline{x}] P \equiv_{\mathfrak{A}} K_{n} \equiv f \bar{a}_{1} \ldots \bar{a}_{q}$ $\equiv g \bar{b}_{1} \ldots \bar{b}_{r} \equiv K_{m+1} \equiv_{\mathfrak{A}} K_{s} \equiv[\underline{V} / \underline{x}] P$, with $K_{j} \not_{1 \mathfrak{A}}^{0} K_{j+1}$ for all $1 \leq j<n$ and $m+\overline{1} \leq j<s$. Hence by Case 1 $U_{i} \equiv_{\mathfrak{A}} V_{i}$ for all $1 \leq i \leq k$.

Lemma 3.1.8. Let $Q$ and $Q^{\prime}$ be terms, $x$ and $y$ be variables. If $y \notin F V(Q)$ and $[y / x] Q \equiv_{1 \mathfrak{A}} Q^{\prime}$, then $Q \equiv_{1 \mathfrak{A}} Q^{\prime \prime}$ for some term $Q^{\prime \prime}$ such that $Q^{\prime \prime} \equiv_{\alpha}[x / y] Q^{\prime}$.

Proof. Assume $y \notin F V(Q)$ and $[y / x] Q \equiv_{1 \mathfrak{A}} Q^{\prime}$. The proof for the case $x \notin F V(Q)$ is easy, so assume $x \in F V(Q)$ and induct on $Q$. We will prove only the case in which $Q$ is a simple abstraction as the proofs of the other cases are straightforward. Assume $Q \equiv \lambda P . Q_{1}$ for some pattern $P$ and some term $Q_{1}$. The proof for the case $y \notin F V(P)$ is easy, so assume $y \in F V(P)$. Then $[y / x] Q \equiv \lambda[z / y] P .[y / x][z / y] Q_{1}$ where $z$ is the first variable not in $F V\left(P Q_{1}\right)$. So $Q^{\prime} \equiv \lambda[z / y] P . Q_{1}^{\prime}$ for some term $Q_{1}^{\prime}$ where $[y / x][z / y] Q_{1} \equiv_{1 \mathfrak{A}} Q_{1}^{\prime}$. By induction, we have $[z / y] Q_{1} \equiv_{1 \mathfrak{A}} Q_{1}^{\prime \prime} \equiv_{\alpha}[x / y] Q_{1}^{\prime}$ for some term $Q_{1}^{\prime \prime}$, and $Q_{1} \equiv_{1 \mathfrak{A}} Q_{1}^{\prime \prime \prime} \equiv_{\alpha}[y / z] Q_{1}^{\prime \prime}$ for some term $Q_{1}^{\prime \prime \prime}$. Note that since $\{x, y\} \cap F V([z / y] P)=\emptyset,[x / y] \lambda[z / y] P \cdot Q_{1}^{\prime} \equiv \lambda[z / y] P .[x / y] Q_{1}^{\prime}$. Choose $Q^{\prime \prime} \equiv \lambda P \cdot Q_{1}^{\prime \prime \prime}$. Then $Q \equiv \lambda P \cdot Q_{1} \equiv_{1 \mathfrak{A}} \lambda P \cdot Q_{1}^{\prime \prime \prime} \equiv_{\alpha} \lambda P \cdot[y / z] Q_{1}^{\prime \prime} \equiv_{\alpha} \lambda P \cdot[y / z][x / y] Q_{1}^{\prime} \equiv_{\alpha} \lambda[z / y] P \cdot[z / y][y / z][x / y] Q_{1}^{\prime} \equiv_{\alpha}$ $\lambda[z / y] P .[x / y] Q_{1}^{\prime} \equiv[x / y] \lambda[z / y] P \cdot Q_{1}^{\prime} \equiv[x / y] Q^{\prime}$.

Lemma 3.1.9. Let $M, N$, and $N^{\prime}$ be terms. If $M \equiv_{1 \alpha} N \equiv_{1 \mathfrak{A}} N^{\prime}$, then $M \equiv_{1 \mathfrak{A}} M^{\prime} \equiv_{\alpha} N^{\prime}$ for some term $M^{\prime}$.

Proof. By induction on $M$.

Corollary 3.1.10. Let $M, N$, and $N^{\prime}$ be terms. If $M \equiv_{\alpha} N \equiv_{\mathfrak{A}} N^{\prime}$ then $M \equiv_{\mathfrak{A}} M^{\prime} \equiv_{\alpha} N^{\prime}$ for some term $M^{\prime}$.

Proof. This follows directly from Lemma 3.1.9.

Lemma 3.1.11. Let $\underline{x}=x_{1}, \ldots, x_{k}, k \geq 1$, be distinct variables and $M, N$, and $\underline{U}=U_{1}, \ldots, U_{k}$ be terms. If $M \equiv_{1 \mathfrak{A}} N$ then $[\underline{U} / \underline{x}] M \equiv_{1 \mathfrak{A}} N^{\prime}$ for some term $N^{\prime}$ such that $N^{\prime} \equiv_{\alpha}[\underline{U} / \underline{x}] N$.

Proof. Assume $M \equiv_{1 \mathfrak{A}} N$. The proof for the case $\{\underline{x}\} \cap F V(M)=\emptyset$ is easy. Assume $\{\underline{x}\} \subseteq F V(M)$ and induct on $M$. We will prove only the case in which $M$ is a simple abstraction as the proofs of the other cases are straightforward. Assume $M \equiv \lambda P . M_{1}$ for some pattern $P$ and some term $M_{1}$. Then $N \equiv \lambda P \cdot N_{1}$ for some term $N_{1}$ such that $M_{1} \equiv_{1 \mathfrak{A}} N_{1}$. By induction $[\underline{U} / \underline{x}] M_{1} \equiv_{1 \mathfrak{A}} N_{1}^{\prime} \equiv_{\alpha}[\underline{U} / \underline{x}] N_{1}$ for some term $N_{1}^{\prime}$. Let $m=|F V(P) \cap F V(\underline{U})|$ and induct on $m$. If $m=0$, then $[\underline{U} / \underline{x}] \bar{M} \equiv \lambda P .[\underline{U} / \underline{x}] M_{1} \equiv_{1 \mathfrak{A}} \lambda P \cdot N_{1}^{\prime} \equiv_{\alpha} \lambda P .[\underline{U} / \underline{x}] N_{1} \equiv[\underline{U} / \underline{x}] N$. Now assume $m>0$. Let $y$ be the first variable in $F V(P) \cap F V(\underline{U})$ and $z$ be the first variable which is not in $F V\left(P M_{1} \underline{U}\right)$. Note that $z$ is also the first variable which is not in $F V\left(P N_{1} \underline{U}\right)$ since $M_{1} \equiv_{1 \mathfrak{A}} N_{1}$, so $F V\left(M_{1}\right) \equiv F V\left(N_{1}\right)$. By the main induction hypothesis, $[z / y] M_{1} \equiv_{1 \mathfrak{A}} N_{1}^{\prime \prime} \equiv_{\alpha}[z / y] N_{1}$ for some term $N_{1}^{\prime \prime}$. Then by the subsidiary induction hypothesis, $[\underline{U} / \underline{x}] \lambda[z / y] P .[z / y] M_{1} \equiv_{1 \mathfrak{A}} N^{\prime} \equiv_{\alpha}[\underline{U} / \underline{x}] \lambda[z / y] P . N_{1}^{\prime \prime}$ for some term $N^{\prime}$. Hence $[\underline{U} / \underline{x}] M \equiv[\underline{U} / \underline{x}] \lambda[z / y] P .[z / y] M_{1} \equiv_{1 \mathfrak{A}} N^{\prime} \equiv_{\alpha}[\underline{U} / \underline{x}] \lambda[z / y] P .[z / y] N_{1} \equiv[\underline{U} / \underline{x}] \lambda P . N_{1} \equiv[\underline{U} / \underline{x}] N$.

Corollary 3.1.12. Let $\underline{x}=x_{1}, \ldots, x_{k}, k \geq 1$, be distinct variables and $M, N$, and $\underline{U}=U_{1}, \ldots, U_{k}$ be terms. If $M \equiv_{\mathfrak{A}} N$ then $[\underline{U} / \underline{x}] M \equiv_{\mathfrak{A}} N^{\prime}$ for some term $N^{\prime}$ such that $N^{\prime} \equiv_{\alpha}[\underline{U} / \underline{x}] N$.

Proof. This follows from Lemma 3.1.11 and Corollary 3.1.10.

\subsection{Contractions and Reductions}

The definitions of $\beta$-contraction, $\gamma$-contraction, and $\delta$-contraction; and potential redex and contractible redex are analogous to those in the original lambda calculus with patterns. We extend the definitions of reductions by adding the new congruence ' $\equiv_{\mathfrak{A}}$ ' as follows.

Definition 3.2.1. For any terms $M$ and $M^{\prime}$, we say $M \beta$-reduces to $M^{\prime}$, denoted by $M \triangleright_{\beta} M^{\prime}$, if there exists a sequence of terms $M \equiv M_{1}, \ldots, M_{n} \equiv M^{\prime}, n \geq 1$, such that for each $1 \leq i<n, M_{i} \triangleright_{1 \beta} M_{i+1}, M_{i} \equiv_{\alpha} M_{i+1}$, or $M_{i} \equiv_{\mathfrak{A}} M_{i+1}$.

Definition 3.2.2. For any terms $M$ and $M^{\prime}$, we say M $\beta \gamma$-reduces to $M^{\prime}$, denoted by $M \triangleright_{\beta \gamma} M^{\prime}$, if there exists a sequence of terms $M \equiv M_{1}, \ldots, M_{n} \equiv M^{\prime}, n \geq 1$, such that for each $1 \leq i<n, M_{i} \triangleright_{1 \beta} M_{i+1}, M_{i} \triangleright_{1 \gamma} M_{i+1}, M_{i} \equiv_{\alpha} M_{i+1}$, or $M_{i} \equiv_{\mathfrak{A}} M_{i+1}$. 
Definition 3.2.3. For any terms $M$ and $M^{\prime}$, we say $M \beta \delta$-reduces to $M^{\prime}$, denoted by $M \triangleright_{\beta \delta} M^{\prime}$, if there exists a sequence of terms $M \equiv M_{1}, \ldots, M_{n} \equiv M^{\prime}, n \geq 1$, such that for each $1 \leq i<n, M_{i} \triangleright_{1 \beta} M_{i+1}, M_{i} \triangleright_{1 \delta} M_{i+1}, M_{i} \equiv_{\alpha} M_{i+1}$, or $M_{i} \equiv_{\mathfrak{A}} M_{i+1}$. We call such a sequence of terms a $\beta \boldsymbol{\delta}$-reduction.

Remark. Unless explicitly specified otherwise, a "reduction" means a“ $\beta \delta$-reduction".

Notation. The expression $M \triangleright_{1 \beta 1 \delta} N$ will mean " $M \triangleright_{1 \beta} N$ or $M \triangleright_{1 \delta} N$ ".

Many of the following lemmas are also analogous to those of the original lambda calculus with patterns. Most of the lemmas are unaffected by the new congruence $\equiv_{\mathfrak{A}}$ and for these proofs will not be given. Only a few need some small changes in the statement or proof; for these we will sketch the proof for those parts that differ.

Corollary 3.2.4. For any term $M$, if $M \triangleright_{\beta \delta} N$, then $N$ is a term and

a. if $M \equiv M_{1} M_{2}$ and $M \triangleright_{\beta \delta} N$ by a sequence of terms $M \equiv K_{1}, \ldots, K_{n} \equiv N, n \geq 1$, such that for each $1 \leq i<n, K_{i}$ is not the potential redex which is contracted and $K_{i} \not_{1 \mathfrak{A}}^{0} K_{i+1}$ then $N \equiv N_{1} N_{2}$ for some terms $N_{1}$ and $N_{2}$ such that $M_{i} \triangleright_{\beta \delta} N_{i}, i=1,2$;

b. if $M \equiv \lambda P . Q$, and no variable in $P$ has been changed when $M \triangleright_{\beta \delta} N$ then $N \equiv \lambda P . Q^{\prime}$ for some term $Q^{\prime}$ such that $Q \triangleright_{\beta \delta} Q^{\prime}$;

c. if $M \equiv(\lambda P . Q \mid A)$ then $N \equiv\left(\lambda P^{\prime} \cdot Q^{\prime} \mid A^{\prime}\right)$ for some abstractions $\lambda P^{\prime} \cdot Q^{\prime}$ and $A^{\prime}$ such that $\lambda P . Q \triangleright_{\beta \delta} \lambda P^{\prime} . Q^{\prime}$ and $A \triangleright_{\beta \delta} A^{\prime}$.

Lemma 3.2.5. Let $\underline{x}=x_{1}, \ldots, x_{k}, k \geq 1$, be distinct variables, $\underline{N}=N_{1}, \ldots, N_{k}$ be terms, and $P$ be a pattern. If $[\underline{N} / \underline{x}] P$ is a potential redex, then $P \equiv x_{t}$ for some $1 \leq t \leq k$.

Lemma 3.2.6. Let $\underline{x}=x_{1}, \ldots, x_{k}, k \geq 1$, be distinct variables, $\underline{N}=N_{1}, \ldots, N_{k}$ be terms, $P$ be a pattern, and $S$ be a potential redex. If $S$ is in $[\underline{N} / \underline{x}] P$, then $S$ is in $N_{t}$ for some $1 \leq t \leq \bar{k}$.

Proof. Assume $S$ is in $[\underline{N} / \underline{x}] P$. We will induct on $P$. Note that since a pattern cannot contain an abstraction, $\{\underline{x}\} \cap F V(P) \neq$ $\emptyset$, otherwise $S$ is in $[\underline{N} / \underline{x}] P \equiv P$, a contradiction. In fact, by Corollary 2.2.1(a) we may assume that $\{\underline{x}\} \subseteq F \bar{V}(P)$.

Case 1. $P \equiv x_{1}$.

Then $[\underline{N} / \underline{x}] P \equiv N_{1}$. So $S$ is in $N_{1}$ and we are finished.

Case 2. $P \equiv P_{1} P_{2}$.

Then $[\underline{N} / \underline{x}] P \equiv[\underline{N} / \underline{x}] P_{1}[\underline{N} / \underline{x}] P_{2}$. Since $P$ is not a variable, by Lemma 3.2.5, any substitution of $P$ is not a potential redex. Hence $S$ is either in $[\underline{N} / \underline{x}] P_{1}$ or $[\underline{N} / \underline{x}] P_{2}$. In either case, by induction $S$ is in $N_{t}$ for some $1 \leq t \leq k$.

Lemma 3.2.7. Let $R \equiv(\lambda P . Q) N$ be a $\beta$-redex, $\underline{x}=x_{1}, \ldots, x_{k}, k \geq 1$, be distinct variables, and $S, \underline{U}=U_{1}, \ldots, U_{k}$ be terms. If $R \triangleright_{1 \beta} S$, then $[\underline{U} / \underline{x}] R \triangleright_{\beta}[\underline{U} / \underline{x}] S$. To be precise, if $R \triangleright_{1 \beta} S$, then $[\underline{U} / \underline{x}] R \triangleright_{1 \beta} S^{*}$ for some term $S^{*}$, where $S^{*} \equiv_{\alpha}[\underline{U} / \underline{x}] S$.

Lemma 3.2.8. Let $R \equiv(\lambda P . Q \mid A) N$ be a $\delta$-redex, $\underline{x}=x_{1}, \ldots, x_{k}, k \geq 1$, be distinct variables, and $\underline{U}=U_{1}, \ldots, U_{k}$ be terms. If $R \triangleright_{1 \delta} S$, then $[\underline{U} / \underline{x}] R \triangleright_{1 \delta}[\underline{U} / \underline{x}] S$.

Lemma 3.2.9. Let $P$ be a pattern with $F V(P)=\left\{x_{1}, \ldots, x_{k}\right\}, k \geq 1$, and $N, \underline{U}=U_{1}, \ldots, U_{k}$ be terms. Let $\underline{x}=x_{1}, \ldots, x_{k}$. If $[\underline{U} / \underline{x}] P \triangleright_{\beta \delta} N$, then $N \equiv_{\mathfrak{A}}[\underline{V} / \underline{x}] P$ for some terms $\underline{V}=V_{1}, \ldots, V_{k}$ such that $U_{i} \triangleright_{\beta \delta} V_{i}$ for all $1 \leq i \leq k$.

Proof. Assume $[\underline{U} / \underline{x}] P \triangleright_{\beta \delta} N$. Induct on $P$. The proof for the case $P \equiv x_{1}$ is easy. Assume $P \equiv P_{1} P_{2}$. Let $[\underline{U} / \underline{x}] P \triangleright_{\beta \delta} N$ by a sequence of terms $[\underline{U} / \underline{x}] P \equiv K_{1}, \ldots, K_{n} \equiv N, n \geq 1$.

Case 1. $K_{i} \not_{1 \mathfrak{A}}^{0} K_{i+1}$ for all $1 \leq i<n$.

Then $N \equiv N_{1} N_{2}$ for some terms $N_{1}$ and $N_{2}$, where $[\underline{U} / \underline{x}] P_{i} \triangleright_{\beta \delta} N_{i}, i=1,2$. Without loss of generality, assume $F V\left(P_{1}\right) \neq \emptyset$.

(1.1) $F V\left(P_{2}\right)=\emptyset$

Then $F V\left(P_{1}\right)=\left\{x_{1}, \ldots, x_{k}\right\}$ and $P_{2} \equiv_{\mathfrak{A}} N_{2}$. By induction $N_{1} \equiv_{\mathfrak{A}}[\underline{V} / \underline{x}] P_{1}$ for some terms $\underline{V}=V_{1}, \ldots, V_{k}$, where $U_{i} \triangleright_{\beta \delta} V_{i}$ for all $1 \leq i \leq k$. Hence $N \equiv_{\mathfrak{A}}\left([\underline{V} / \underline{x}] P_{1}\right) P_{2} \equiv[\underline{V} / \underline{x}] P$.

(1.2) $F V\left(P_{2}\right)=\left\{x_{j_{1}}, \ldots, x_{j_{p}}\right\}$.

Then $F V\left(P_{1}\right)=\left\{x_{i_{1}}, \ldots, x_{i_{m}}\right\}$, where $\left\{i_{1}, \ldots, i_{m}\right\} \cup\left\{j_{1}, \ldots, j_{p}\right\}=\{1, \ldots, k\}$ and $\left\{i_{1}, \ldots, i_{m}\right\} \cap\left\{j_{1}, \ldots, j_{p}\right\}=$ $\emptyset$. By Corollary 2.2.1(a), $[\underline{U} / \underline{x}] P_{1} \equiv\left[U_{i_{1}} / x_{i_{1}}, \ldots, U_{i_{m}} / x_{i_{m}}\right] P_{1}$ and $[\underline{U} / \underline{x}] P_{2} \equiv\left[U_{j_{1}} / x_{j_{1}}, \ldots, U_{j_{p}} / x_{j_{p}}\right] P_{2}$. By induction, $N_{1} \equiv_{\mathfrak{A}}\left[V_{i_{1}} / x_{i_{1}}, \ldots, V_{i_{m}} / x_{i_{m}}\right] P_{1}$ and $N_{2} \equiv_{\mathfrak{A}}\left[V_{j_{1}} / x_{j_{1}}, \ldots, V_{j_{p}} / x_{j_{p}}\right] P_{2}$ for some terms $V_{i_{1}}, \ldots, V_{i_{m}}, V_{j_{1}}, \ldots, V_{j_{p}}$, where $U_{r} \triangleright_{\beta \delta} V_{r}$ for all $1 \leq r \leq k$. Let $\underline{V}=V_{1}, \ldots, V_{k}$. Hence $N \equiv N_{1} N_{2} \equiv_{\mathfrak{A}}$ $\left[V_{i_{1}} / x_{i_{1}}, \ldots, V_{i_{m}} / x_{i_{m}}\right] P_{1}\left[V_{j_{1}} / x_{j_{1}}, \ldots, V_{j_{p}} / x_{j_{p}}\right] P_{2} \equiv[\underline{V} / \underline{x}] P_{1}[\underline{V} / \underline{x}] P_{2} \equiv[\underline{V} / \underline{x}] P$.

Case 2. $K_{i} \equiv_{1 \mathfrak{A}}^{0} K_{i+1}$ for some $1 \leq i<n$.

Let $k$ be the first such $i$. Then $[\underline{U} / \underline{x}] P \triangleright_{\beta \delta} K_{k}$ with $K_{j} \not_{1 \mathfrak{A}}^{0} K_{j+1}$ for all $1 \leq j<k$. By Case 1 we have $K_{k} \equiv_{\mathfrak{A}}[\underline{V} / \underline{x}] P$ for some terms $\underline{V}=V_{1}, \ldots, V_{k}$ such that $U_{j} \triangleright_{\beta \delta} V_{j}$ for all $1 \leq j \leq k$. Since $K_{k} \equiv_{1 \mathfrak{A}}^{0} K_{k+1}, K_{k}$ contains no abstraction, so $N \equiv_{\mathfrak{A}} K_{k} \equiv_{\mathfrak{A}}[\underline{V} / \underline{x}] P$.

Lemma 3.2.10. Let $A$ be an abstraction, and $N$ be a term such that $A N$ is a contractible redex. 
a. For any term $N^{\prime}$ such that $N \equiv_{\alpha} N^{\prime}, A N^{\prime}$ is a contractible redex.

b. For any term $N^{\prime}$ such that $N \triangleright_{1 \beta 1 \delta} N^{\prime}, A N^{\prime}$ is a contractible redex.

Lemma 3.2.11. Let $A$ be an abstraction, and $N$ and $N^{\prime}$ be terms such that $N \triangleright_{\beta \delta} N^{\prime}$. If $A N$ is a contractible redex, then there exists a term $N^{\prime \prime}$ such that $N^{\prime} \equiv_{\mathfrak{A}} N^{\prime \prime}$ and $A N^{\prime \prime}$ is a contractible redex.

Proof. Assume $A N$ is contractible. Let $N \triangleright_{\beta \delta} N^{\prime}$ by a sequence of terms $N \equiv N_{1}, \ldots, N_{k} \equiv N^{\prime}, k \geq 1$. We will induct on $k$. If $k=1$ then $N \equiv N_{1} \equiv N^{\prime}$, so $A N^{\prime}$ is contractible. Now assume $k>1$. Then by induction there exists a term $N_{k-1}^{\prime}$ such that $N_{k-1} \equiv_{\mathfrak{A}} N_{k-1}^{\prime}$ and $A N_{k-1}^{\prime}$ is a contractible redex. We will prove only the case in which $N_{k-1} \triangleright_{1 \beta 1 \delta} N^{\prime}$ as the proofs of the other cases are straightforward. Assume $N_{k-1} \triangleright_{1 \beta 1 \delta} N^{\prime}$. Then $N_{k-1}$ contains an abstraction. Since $N_{k-1} \equiv_{\mathfrak{A}} N_{k-1}^{\prime}$, by Note 3.1.4(a), $N_{k-1}^{\prime}$ also contains an abstraction.

Case 1. $A \equiv \lambda P$. $Q$. for some pattern $P$ and some term $Q$.

Assume $F V(P)=\left\{x_{1}, \ldots, x_{n}\right\}$ for some variables $\underline{x}=x_{1}, \ldots, x_{n}$. Since $(\lambda P . Q) N_{k-1}^{\prime}$ is contractible, $[\underline{U} / \underline{x}] P \equiv$ $N_{k-1}^{\prime}$ for some terms $\underline{U}=U_{1}, \ldots, U_{n}$. Since $[\underline{U} / \underline{x}] P \equiv N_{k-1}^{\prime} \equiv_{\mathfrak{A}} N_{k-1} \triangleright_{1 \beta 1 \delta} N^{\prime}$, by Lemma $3.2 .9 N^{\prime} \equiv \equiv_{\mathfrak{A}}[\underline{V} / \underline{x}] P$ for some terms $\underline{V}=\overline{V_{1}}, \ldots, V_{n}$. Choose $N^{\prime \prime} \equiv[\underline{V} / \underline{x}] P$.

Case 2. $A \equiv(\lambda P . Q \mid B)$ for some pattern $P$, some term $\bar{Q}$, and some abstraction $B$.

If $A N_{k-1}$ is contractible, since $N_{k-1} \triangleright_{1 \beta 1 \delta} N^{\prime}$, by Lemma 3.2.10(b), $A N^{\prime}$ is contractible and we are finished. Now suppose $(\lambda P . Q \mid B) N_{k-1}$ is not contractible. Then $(\lambda P . Q \mid B) N_{k-1}^{\prime} \beta_{1 \delta} B N_{k-1}^{\prime}$, so $(\lambda P . Q) N_{k-1}^{\prime}$ is contractible, and the proof can be finished much like in Case 1.

Lemma 3.2.12. Let $R$ be a contractible redex, and $R^{\prime}$ and $S$ be terms such that $R \equiv_{\alpha} R^{\prime}$. If $R \triangleright_{1 \beta} S$ (respectively $R \triangleright_{1 \delta} S$ ), then $R^{\prime} \triangleright_{1 \beta} S^{\prime}$ (respectively $R^{\prime} \triangleright_{1 \delta} S^{\prime}$ ) for some term $S^{\prime}$, where $S^{\prime} \equiv_{\alpha} S$.

\subsection{Computability Relative to a Structure}

Definition 3.3.1. Let $g$ be an $n$-ary function on $|\mathfrak{A}|$. We say $g$ is computable relative to $\mathfrak{A}$ if and only if there is a term $G$, using only variables and symbols in $\mathcal{L}$ together with $\mathrm{T}$ and $\mathrm{F}$, such that for all $a_{1}, \ldots, a_{n}, a \in|\mathfrak{A}|$, we have

$$
G \bar{a}_{1} \ldots \bar{a}_{n} \triangleright_{\beta \delta} \bar{a}
$$

whenever $g\left(a_{1}, \ldots, a_{n}\right)=a$.

Definition 3.3.2. Let $r$ be an $n$-ary relation on $|\mathfrak{A}|$. We say $r$ is computable relative to $\mathfrak{A}$ if and only if there is a term $R$, using only variables and symbols in $\mathcal{L}$ together with $\mathrm{T}$ and $\mathrm{F}$, such that for all $a_{1}, \ldots, a_{n} \in|\mathfrak{A}|$, we have

$$
\begin{aligned}
& R \bar{a}_{1} \ldots \bar{a}_{n} \triangleright_{\beta \delta} \mathrm{T} \text { if }\left(a_{1}, \ldots, a_{n}\right) \in r, \text { and } \\
& R \bar{a}_{1} \ldots \bar{a}_{n} \triangleright_{\beta \delta} \mathrm{F} \text { otherwise. }
\end{aligned}
$$

\section{The Church-Rosser Theorem}

\subsection{Minimal Complete Developments}

The definition for minimal complete development $(M C D)$ is slightly modified from the original one to allow the new congruence $\equiv_{\mathfrak{A}}$.

Definition 4.1.1. Let $R$ and $S$ be occurrences of contractible redexes in a term $M$. When $R$ is contracted, let $M$ change to $M^{\prime}$.

The contraction-residuals of $S$ (with respect to $R$ ) are occurrences of potential redexes in $M^{\prime}$, defined as follows.

Case 1. $R$ and $S$ are non-overlapping parts of $M$.

Then contracting $R$ leaves $S$ unchanged. This unchanged $S$ in $M^{\prime}$ is the contraction-residual of $S$.

Case 2. $R \equiv S$

Then contracting $R$ is the same as contracting $S$. We say $S$ has no contraction-residuals in $M^{\prime}$.

Case 3. $R$ is part of $S$ and $R \not \equiv S$.

Since $S$ is a potential redex, $S \equiv A N$ for some abstraction $A$, and some term $N$. So $R$ is either in $A$ or in $N$. Then contracting $R$ changes $S$ to $S^{\prime}$, where $S^{\prime} \equiv A^{\prime} N^{\prime}$ for some abstraction $A^{\prime}$ and some term $N^{\prime}$ such that either $A \triangleright_{1 \beta 1 \delta} A^{\prime}$ and $N \equiv N^{\prime}$ or $A \equiv A^{\prime}$ and $N \triangleright_{1 \beta 1 \delta} N^{\prime}$. This $S^{\prime}$ is the contraction-residual of $S$.

Case 4. $S$ is part of $R$ and $S \not \equiv R$.

There are cases and subcases as follows.

(4.1) $R \equiv(\lambda P . Q) N$.

(4.1.1) $F V(P)=\emptyset$.

Since $R$ is a $\beta$-redex, $P \equiv N$ and $R \triangleright_{1 \beta} Q$. Since $S$ is a potential redex in $R, S$ is in $Q$. Since $R \triangleright_{1 \beta} Q$, contracting $R$ leaves $S$ unchanged in $M^{\prime}$; this is the contraction-residual of S. 
(4.1.2) $F V(P)=\left\{x_{1}, \ldots, x_{k}\right\}, k \geq 1$.

Then $[\underline{N} / \underline{x}] P \equiv N$ for some terms $\underline{N}=N_{1}, \ldots, N_{k}$ and $R \triangleright_{1 \beta}[\underline{N} / \underline{x}] Q$.

(4.1.2.1) $S$ is in $Q$.

Then $S$ changes to $S^{\prime}$, where $S^{\prime}$ is either $S$ or some substitution of $S$. This $S^{\prime}$ is the contractionresidual of $S$.

(4.1.2.2) $S$ is in $N$.

Then $S$ is in $[N / x] P$. By Lemma 3.2.6, $S$ is in $N_{t}$ for some $1 \leq t \leq k$. Hence there is an occurrence of $S$ in each $N_{t}$ substituted for an occurrence of $x_{t}$ in $Q$. These are the contractionresiduals of $S$. (Note that $S$ may have many or no contraction-residuals.)

(4.2) $R \equiv(\lambda P . Q \mid A) N$.

(4.2.1) $R \triangleright_{1 \delta}(\lambda P . Q) N$.

If $S$ is in $Q$ or $N$, then contracting $R$ leaves $S$ unchanged, and this is the contraction-residual of $S$ in $M^{\prime}$. If $S$ is in $A$, then $S$ has no contraction-residuals in $M^{\prime}$.

(4.2.2) $R \triangleright_{1 \delta} A N$.

If $S$ is in $A$ or $N$, then this unchanged $S$ in $A$ or $N$ is the contraction-residual of $S$ in $M^{\prime}$. If $S$ is in $Q$, then $S$ has no contraction-residuals in $M^{\prime}$.

\section{Note 4.1.2.}

a. Except in the Case 4.1.2.2, $S$ has at most one contraction-residual.

b. Each contraction-residual is a contractible redex.

Definition 4.1.3. Let $R$ be an occurrence of contractible redex in a term $M$. The 12 -conversion-residuals of $R$ (with respect to $M$ ) when $M \equiv_{1 \mathfrak{A}} M^{\prime}$ are occurrences of potential redexes in $M^{\prime}$, defined inductively as follows. Note that since $M$ contains an abstraction, $M \neq_{1 \mathfrak{A}}^{0} M^{\prime}$, so $M$ and $M^{\prime}$ are of the same form.

Case $1 . M \equiv R$.

If $M^{\prime}$ is a contractible redex then this $M^{\prime}$ is the $1 \mathfrak{A}$-conversion-residual of $R$, otherwise $R$ has no $1 \mathfrak{A}$-conversionresiduals in $M^{\prime}$.

Case 2. $M \not \equiv R$.

(2.1) $R$ is unchanged in $M^{\prime}$.

This unchanged $R$ is the $1 \mathfrak{A}$-conversion-residual of $R$.

(2.2) $R$ is changed in $M^{\prime}$.

(2.2.1) $M \equiv M_{1} M_{2}$ for some term $M_{1}$ and $M_{2}$.

Then $R$ is in $M_{i}$ for some $i \in\{1,2\}$. The $1 \mathfrak{A}$-conversion-residual of $R$ with respect to $M_{i}$ is the $1 \mathfrak{A}$-conversion-residual of $R$ with respect to $M$.

(2.2.2) $M \equiv \lambda P . N$ for some pattern $P$ and some term $N$.

Then $R$ is in $N$. The $1 \mathfrak{A}$-conversion-residual of $R$ with respect to $N$ is the $1 \mathfrak{A}$-conversion-residual of $R$ with respect to $M$.

(2.2.3) $M \equiv\left(A_{1} \mid A_{2}\right)$ for some abstractions $A_{1}$ and $A_{2}$.

Then $R$ is in $A_{i}$ for some $i \in\{1,2\}$. The $1 \mathfrak{A}$-conversion-residual of $R$ with respect to $A_{i}$ is the $1 \mathfrak{A}$-conversion-residual of $R$ with respect to $M$.

Note 4.1.4.

a. $R$ has at most one $1 \mathfrak{A}$-conversion-residual.

b. Each $1 \mathfrak{A}$-conversion-residual is a contractible redex.

Remark. We may simply use "residual" to abbreviate either a "contraction-residual" or a "12-conversion-residual", where there is no ambiguity.

Definition 4.1.5. If $\mathscr{R}=\left\{R_{i} \mid 1 \leq i \leq n\right\}, n \geq 0$, is a set of occurrences of potential redexes in a term $M$, then an $R_{i}$ is called minimal (with respect to $\mathscr{R}$ ) if it properly contains no other $R_{j} \in \mathscr{R}$.

Let $\mathscr{R}_{M}=\left\{R_{i} \mid 1 \leq i \leq n\right\}, n \geq 0$, be a set of occurrences of contractible redexes in a term $M$. For any terms $M^{*}$ and $M^{\prime}$ such that $M^{*} \equiv_{\mathfrak{A}} M$, we say $M^{\prime}$ is obtained from $M^{*}$ by a minimal complete development (MCD) of $\mathscr{R}_{M}$, denoted by $M^{*} \triangleright_{m c d} M^{\prime}$ (of $\mathscr{R}_{M}$ ), if $M^{\prime}$ is obtained from $M$ by the following process.

First contract any minimal $R_{i}$; without loss of generality let $i=1$. By Definition 4.1.1, this leaves $n-1$ contractionresiduals, $R_{2}^{\prime}, R_{3}^{\prime}, \ldots, R_{n}^{\prime}$. Then make as many $1 \mathfrak{A}$-conversions as you like (possibly none), this leaves at most $n-1$ $1 \mathfrak{A}$-conversion-residuals among $R_{2}^{\prime \prime}, R_{3}^{\prime \prime}, \ldots, R_{n}^{\prime \prime}$. Again, contract any minimal $R_{t}^{\prime \prime}$ and make $1 \mathfrak{A}$-conversions. This leaves at most $n-2$ residuals. Repeat this process until no contraction-residuals are left. Then make as many $1 \mathfrak{A}$-conversions as you like. Finally, make as many $\alpha$-steps as you like. 
Note 4.1.6. a. Each MCD is a $\beta \delta$-reduction.

b. For any contractible redex $L$, if $L \triangleright_{m c d} M$ of $\mathscr{R}_{L}$, without $\alpha$-steps, where $L \notin \mathscr{R}_{L}$, and $M \triangleright_{1 \beta 1 \delta} N$, with $M$ being the potential redex contracted, then $L \triangleright_{m c d} N$ of $\mathscr{R}_{L} \cup\{L\}$, without $\alpha$-steps. In fact, for any term $L^{\prime}$ such that $L^{\prime} \equiv_{\mathfrak{A}} L$, $L^{\prime} \triangleright_{m c d} N$ of $\mathscr{R}_{L} \cup\{L\}$, without $\alpha$-steps.

Proposition 4.1.7. Let $M, N$ and $N^{\prime}$ be terms. If $M \triangleright_{m c d} N \equiv_{\mathfrak{A}} N^{\prime}$ then $M \triangleright_{m c d} N^{\prime}$.

Proof. This follows directly from Corollary 3.1.10.

Lemma 4.1.8. Let $P$ be a pattern with $F V(P)=\left\{x_{1}, \ldots, x_{k}\right\}, k \geq 1$, and $N, \underline{U}=U_{1}, \ldots$, $U_{k}$ be terms. Let $\underline{x}=x_{1}, \ldots, x_{k}$. If $[\underline{U} / \underline{x}] P \triangleright_{m c d} N$, then $N \equiv_{\mathfrak{A}}[\underline{V} / \underline{x}] P$ for some terms $\underline{V}=V_{1}, \ldots, V_{k}$ such that $U_{i} \triangleright_{m c d} V_{i}$ for all $1 \leq i \leq k$.

Proof. This can be proved in the same way as Lemma 3.2.9.

Lemma 4.1.9. For any terms $M, N$, and $M^{\prime}$, if $M \triangleright_{m c d} N$ and $M \equiv_{\alpha} M^{\prime}$, then $M^{\prime} \triangleright_{m c d} N$.

Lemma 4.1.10. For any distinct variables $\underline{x}=x_{1}, \ldots, x_{k}, k \geq 1$, and any terms $M, N, \underline{U}=U_{1}, \ldots, U_{k}, \underline{V}=V_{1}, \ldots, V_{k}$ if $M \triangleright_{m c d} N$ and $U_{i} \triangleright_{m c d} V_{i}$ for all $1 \leq i \leq k$, then $[\underline{U} / \underline{x}] M \triangleright_{m c d}[\underline{V} / \underline{x}] N$.

Proof. As in the original proof of these two lemmas (Vejjajiva, 1997, pp. 56-59), they are proved simultaneously by induction on $M$, and additionally we may assume that the MCD $M \triangleright_{m c d} N$ has no $\alpha$-steps and $\{\underline{x}\} \subseteq F V(M)$. The proof remains unchanged except for the case where $M \equiv M_{1} M_{2}$, which is rewritten as follows. Let $M \triangleright_{m c d} N$ of $\mathscr{R}$ by a sequence of terms $M \equiv K_{1}, \ldots, K_{n} \equiv N, n \geq 1$.

Case $1 . K_{i} \not_{1 \mathfrak{A}}^{0} K_{i+1}$ for all $1 \leq i<n$.

(1.1) $M \notin \mathscr{R}$.

This can be proved in the same way as the case when $M$ is a compound abstraction (See Case iii. of the original proof (Vejjajiva, 1997, pp.58).

(1.2) $M \in \mathscr{R}$.

Then $M \triangleright_{m c d} M_{1}^{0} M_{2}^{0} \triangleright_{1 \beta 1 \delta} N^{0} \equiv_{\mathfrak{A}} N$ for some terms $M_{1}^{0}, M_{2}^{0}$, and $N^{0}$ such that $M_{1} \triangleright_{m c d} M_{1}^{0}$ and $M_{2} \triangleright_{m c d} M_{2}^{0}$, both without $\alpha$-steps, and $M_{1}^{0} M_{2}^{0}$ is the potential redex contracted when $M_{1}^{0} M_{2}^{0} \triangleright_{1 \beta 1 \delta} N^{0}$.

Proof of 4.1.9.

Then $M^{\prime} \equiv M_{1}^{\prime} M_{2}^{\prime}$ for some terms $M_{1}^{\prime}$ and $M_{2}^{\prime}$ such that $M_{i}^{\prime} \equiv_{\alpha} M_{i}, i=1,2$. By induction, $M_{i}^{\prime} \triangleright_{m c d} M_{i}^{*}$, without $\alpha$-steps, for some terms $M_{i}^{*}$ such that $M_{i}^{*} \equiv_{\alpha} M_{i}^{0}, i=1,2$. Since $M_{1}^{*} M_{2}^{*} \equiv_{\alpha}$ $M_{1}^{0} M_{2}^{0} \triangleright_{1 \beta 1 \delta} N^{0}$, by Lemma 3.2.12 $M_{1}^{*} M_{2}^{*} \triangleright_{1 \beta 1 \delta} M^{*} \equiv_{\alpha} N^{0}$ for some term $M^{*}$. Since $M^{\prime} \equiv$ $M_{1}^{\prime} M_{2}^{\prime} \triangleright_{m c d} M_{1}^{*} M_{2}^{*} \triangleright_{1 \beta 1 \delta} M^{*} \equiv_{\alpha} N^{0} \equiv_{\mathfrak{A}} N$, by Note 4.1.6(b) and Proposition 4.1.7 $M^{\prime} \triangleright_{m c d} N$.

Proof of 4.1.10.

By induction $[\underline{U} / x] M_{i} \triangleright_{m c d} M_{i}^{*}$, without $\alpha$-steps, for some $M_{i}^{*}$ such that $M_{i}^{*} \equiv_{\alpha}[\underline{V} / x] M_{i}^{0}, i=$ 1,2. Since $M_{1}^{0} M_{2}^{0} \triangleright_{1 \beta 1 \delta} N^{0}$, by Lemmas 3.2.7 and 3.2.8 $[\underline{V} / \underline{x}]\left(M_{1}^{0} M_{2}^{0}\right) \triangleright_{1 \beta 1 \delta} N^{*} \equiv_{\alpha}[\underline{V} / \underline{x}] N^{0}$ for some term $N^{*}$. By Lemma 3.2.12 $M_{1}^{*} M_{2}^{*} \triangleright_{1 \beta 1 \delta} M^{*} \equiv_{\alpha} N^{*}$ for some term $M^{*}$. Since $N^{0} \equiv_{\mathfrak{A}}$ $N$, by Proposition $3.1 .12[\underline{V} / \underline{x}] N^{0} \equiv_{\mathfrak{A}} N^{\prime} \equiv_{\alpha}[\underline{V} / \underline{x}] N$ for some term $N^{\prime}$. Since $[\underline{U} / \underline{x}] M \equiv$ $[\underline{U} / \underline{x}] M_{1}[\underline{U} / \underline{x}] M_{2} \triangleright_{m c d} M_{1}^{*} M_{2}^{*} \triangleright_{1 \beta 1 \delta} M^{*} \equiv_{\alpha} N^{*} \equiv_{\alpha}[\underline{V} / \underline{x}] N^{0} \equiv_{\mathfrak{A}} N^{\prime} \equiv_{\alpha}[\underline{V} / \underline{x}] N$, by Note 4.1.6(b) and Proposition 4.1.7, $\left[\underline{U} / \underline{x]} M \triangleright_{m c d}[\underline{V} / \underline{x}] N\right.$.

Case 2. $K_{i} \equiv_{1 \mathfrak{A}}^{0} K_{i+1}$ for some $1 \leq i<n$.

Let $k$ be the first such $i$. Then $M \triangleright_{m c d} K_{k}$ with $K_{j} \not_{1 \mathfrak{A}}^{0} K_{j+1}$ for all $1 \leq j<k$. Since $K_{k} \equiv_{1 \mathfrak{A}}^{0} K_{k+1}, K_{k}$ contains no abstractions. Then, since $K_{k} \triangleright_{m c d} N$, it must be that $K_{k} \equiv_{\mathfrak{A}} N$.

Proof of 4.1.9.

Since $M \equiv_{\alpha} M^{\prime}$, by Case $1, M^{\prime} \triangleright_{m c d} K_{k}$, then by Proposition 4.1.7, $M^{\prime} \triangleright_{m c d} N$.

Proof of 4.1.10.

By Case $1,[\underline{U} / \underline{x}] M \triangleright_{m c d}[\underline{V} / \underline{x}] K_{k}$. Since $K_{k} \equiv_{\mathfrak{A}} N$, by Proposition 3.1.12 $[\underline{V} / \underline{x}] K_{k} \equiv_{\mathfrak{A}} N^{\prime} \equiv_{\alpha}[\underline{V} / \underline{x}] N$ for some term $N^{\prime}$, so by Proposition 4.1.7 $[\underline{U} / \underline{x}] M \triangleright_{m c d}[\underline{V} / \underline{x}] N$.

\subsection{The Church-Rosser Theorem for $\beta \delta$-Reduction}

As is now standard, we first prove the Church-Rosser theorem for MCD's, where most of the work is done, then use it to prove the Church-Rosser theorem for $\beta \delta$-reduction.

Theorem 4.2.1. (The Church-Rosser Theorem for MCD's)

For any terms $L, M$, and $N$, if $L \triangleright_{m c d} M$ and $L \triangleright_{m c d} N$, then there exists a term $T$ such that $M \triangleright_{m c d} T$ and $N \triangleright_{m c d} T$.

Proof. Let $L, M$, and $N$ be terms such that $L \triangleright_{m c d} M$ and $L \triangleright_{m c d} N$. Then $M$ (respectively $N$ ) is obtained from $L$ by the given MCD of a set $\mathscr{R}_{M}$ (respectively $\mathscr{R}_{N}$ ). By Lemma 4.1.9, it is sufficient to consider the case in which the given MCD's have no $\alpha$-steps. Induct on $L$. We will prove only the case in which $L$ is an application as the proofs of the other cases are straightforward. Let $L \equiv L_{1} L_{2}$. The cases $L \notin \mathscr{R}_{M}$ and $L \notin \mathscr{R}_{N}$ can be proved using arguments similar to the one used in the case $L$ is a compound abstraction. Suppose $L \in \mathscr{R}_{M}$. There are cases and subcases as follows. 
Case 1. $L_{1} \equiv \lambda P . Q$.

Then $L \triangleright_{m c d}\left(\lambda P . Q^{M}\right) L_{2}^{M}$ for some terms $Q^{M}$ and $L_{2}^{M}$ such that $Q \triangleright_{m c d} Q^{M}, L_{2} \triangleright_{m c d} L_{2}^{M}$, and $\left(\lambda P . Q^{M}\right) L_{2}^{M} \triangleright_{1 \beta} M^{0} \equiv_{\mathfrak{A}}$ $M$, with $\left(\lambda P \cdot Q^{M}\right) L_{2}^{M}$ being the $\beta$-redex contracted, for some term $M^{0}$. The case $F V(P)=\emptyset$ is easy. Suppose $F V(P)=\left\{x_{1}, \ldots, x_{k}\right\}$. Then there exist terms $\underline{U}=U_{1}, \ldots, U_{k}$, such that $[\underline{U} / \underline{x}] P \equiv L_{2}^{M}$, and $M^{0} \equiv[\underline{U} / \underline{x}] Q^{M}$.

(1.1) $L \in \mathscr{R}_{N}$.

Then there exist terms $Q^{N}, L_{2}^{N}, N^{0}$, and $\underline{V}=V_{1}, \ldots, V_{k}$ for $N$, analogous to $Q^{M}, L_{2}^{M}, M^{0}$, and $\underline{U}$, respectively, for $M$. By induction, there exist terms $Q^{*}$ and $L_{2}^{*}$ such that $Q^{M} \triangleright_{m c d} Q^{*}, Q^{N} \triangleright_{m c d} Q^{*}$, $L_{2}^{M} \triangleright_{m c d} L_{2}^{*}$, and $L_{2}^{N} \triangleright_{m c d} L_{2}^{*}$. Since $L_{2}^{M} \triangleright_{m c d} L_{2}^{*}$ and $L_{2}^{N} \triangleright_{m c d} L_{2}^{*}$, by Lemma 4.1.8 $L_{2}^{*} \equiv_{\mathfrak{A}}\left[\underline{U}^{\prime} / \underline{x}\right] P$ and $L_{2}^{*} \equiv_{\mathfrak{A}}\left[\underline{V}^{\prime} / \underline{x}\right] P$ for some terms $\underline{U^{\prime}}=U_{1}^{\prime}, \ldots, U_{k}^{\prime}, \underline{V}^{\prime}=V_{1}^{\prime}, \ldots, V_{k}^{\prime}$ such that $U_{i} \triangleright_{m c d} U_{i}^{\prime}$, and $V_{i} \triangleright_{m c d} V_{i}^{\prime}$ for all $1 \leq \bar{i} \leq \bar{k}$. Since $\left[\underline{U}^{\prime} / \underline{x}\right] P \equiv_{\mathfrak{A}} L_{2}^{*} \equiv_{\mathfrak{A}}\left[\underline{V}^{\prime} / \underline{x}\right] P$, by Lemma 3.1.7, for each $1 \leq i \leq k, U_{i}^{\prime} \equiv_{\mathfrak{A}} V_{i}^{\prime}$. Since $V_{i} \triangleright_{m c d} V_{i}^{\prime} \equiv_{\mathfrak{A}} U_{i}^{\prime}$, by Proposition 4.1.7, $V_{i} \triangleright_{m c d} U_{i}^{\prime}$ for all $1 \leq i \leq k$. Choose $T \equiv\left[\underline{U^{\prime}} / \underline{x}\right] Q^{*}$.

(1.2) $L \notin \mathscr{R}_{N}$.

Then $N \equiv_{\mathfrak{A}}\left(\lambda P \cdot Q^{N}\right) L_{2}^{N}$ for some terms $Q^{N}$ and $L_{2}^{N}$ such that $Q \triangleright_{m c d} Q^{N}$ and $L_{2} \triangleright_{m c d} L_{2}^{N}$. By induction, there exist terms $Q^{*}$ and $L_{2}^{*}$ such that $Q^{N} \triangleright_{m c d} Q^{*}$ and $L_{2}^{N} \triangleright_{m c d} L_{2}^{*}$, both without $\alpha$-steps, and $Q^{M} \triangleright_{m c d} Q^{*}$ and $L_{2}^{M} \triangleright_{m c d} L_{2}^{*}$. By Lemma 4.1.8, $L_{2}^{*} \equiv_{\mathfrak{A}}[\underline{V} / \underline{x}] P$ for some terms $\underline{V}=V_{1}, \ldots, V_{k}$ such that $U_{i} \triangleright_{m c d} V_{i}$ for all $1 \leq i \leq k$. Since $L \equiv(\lambda P . Q) L_{2}$ is contractible, there exist terms $W=W_{1}, \ldots, W_{k}$ such that $[\underline{W} / \underline{x}] P \equiv L_{2}$. Since $L_{2} \triangleright_{m c d} L_{2}^{N} \triangleright_{m c d} L_{2}^{*}$, by Lemma 4.1.8, $L_{2}^{N} \equiv_{\mathfrak{A}}\left[\underline{W}^{\prime} / \underline{x}\right] P$ and $L_{2}^{*} \equiv_{\mathfrak{A}}\left[\underline{W^{\prime \prime}} / \underline{x}\right] P$ for some terms $\underline{W^{\prime}}=W_{1}^{\prime}, \ldots, W_{k}^{\prime}$, and $\underline{W}^{\prime \prime}=W_{1}^{\prime \prime}, \ldots, W_{k}^{\prime \prime}$ such that $W_{i} \triangleright_{m c d} W_{i}^{\prime} \triangleright_{m c d} W_{i}^{\prime \prime}$ for all $1 \leq i \leq k$. Since $\left[\underline{W}^{\prime \prime} / \underline{x}\right] P \equiv_{\mathfrak{A}} L_{2}^{*} \equiv_{\mathfrak{A}}[\underline{V} / \underline{x}] P, \overline{b y}$ Lemma 3.1.7, $W_{i}^{\prime \prime} \equiv_{\mathfrak{A}} V_{i}$ for all $1 \leq i \leq k$. Choose $T \equiv[\underline{V} / \underline{x}] Q^{*}$.

Case 2. $L_{1} \equiv(\lambda P . Q \mid A)$.

Then $L \triangleright_{m c d}\left(\lambda P . Q^{M} \mid A^{M}\right) L_{2}^{M}$ for some terms $Q^{M}$ and $L_{2}^{M}$, and some abstraction $A^{M}$ such that $Q \triangleright_{m c d} Q^{M}$, $A \triangleright_{m c d} A^{M}, L_{2} \triangleright_{m c d} L_{2}^{M}$, and $\left(\lambda P . Q^{M} \mid A^{M}\right) L_{2}^{M} \triangleright_{1 \delta} M^{0} \equiv_{\mathfrak{A}} M$, with $\left(\lambda P . Q^{M} \mid A^{M}\right) L_{2}^{M}$ being the $\delta$-redex contracted, for some term $M^{0}$.

(2.1) $L \in \mathscr{R}_{N}$.

Then there exist terms $Q^{N}, L_{2}^{N}, A^{N}$, and $N^{0}$, analogous to $Q^{M}, L_{2}^{M}, A^{M}$, and $M^{0}$, for $M$. By induction, there exist terms $Q^{*}, A^{*}$ and $L_{2}^{*}$ such that $Q^{M} \triangleright_{m c d} Q^{*}, Q^{N} \triangleright_{m c d} Q^{*}, A^{M} \triangleright_{m c d} A^{*}, A^{N} \triangleright_{m c d} A^{*}, L_{2}^{M} \triangleright_{m c d} L_{2}^{*}$, and $L_{2}^{N} \triangleright_{m c d} L_{2}^{*}$.

(2.1.1) $\left(\lambda P . Q^{M} \mid A^{M}\right) L_{2}^{M} \triangleright_{1 \delta}\left(\lambda P . Q^{M}\right) L_{2}^{M}$.

Then by Lemma 3.2.11, $\left(\lambda P . Q^{N} \mid A^{N}\right) L_{2}^{N} \triangleright_{1 \delta}\left(\lambda P \cdot Q^{N}\right) L_{2}^{N}$. Choose $T \equiv\left(\lambda P . Q^{*}\right) L_{2}^{*}$.

(2.1.2) $\left(\lambda P . Q^{M} \mid A^{M}\right) L_{2}^{M} \triangleright_{1 \delta} A^{M} L_{2}^{M}$.

By contradiction, using an argument similar to the above, we have that $\left(\lambda P \cdot Q^{N}\right) L_{2}^{N}$ is not a $\beta$-redex, so $\left(\lambda P . Q^{N} \mid A^{N}\right) L_{2}^{N} \triangleright_{1 \delta} A^{N} L_{2}^{N}$. Choose $T \equiv A^{*} L_{2}^{*}$.

(2.2) $L \notin \mathscr{R}_{N}$.

Then $N \equiv_{\mathfrak{A}}\left(\lambda P \cdot Q^{N} \mid A^{N}\right) L_{2}^{N}$ for some terms $Q^{N}$ and $L_{2}^{N}$ and some abstraction $A^{N}$ such that $Q \triangleright_{m c d}$ $Q^{N}, A \triangleright_{m c d} A^{N}$, and $L_{2} \triangleright_{m c d} L_{2}^{N}$. By induction, there exist terms $Q^{*}, A^{*}$, and $L_{2}^{*}$, such that $Q^{N} \triangleright_{m c d} Q^{*}, A^{N} \triangleright_{m c d}$ $A^{*}$, and $L_{2}^{N} \triangleright_{m c d} L_{2}^{*}$, all without $\alpha$-steps, and $Q^{M} \triangleright_{m c d} Q^{*}, A^{M} \triangleright_{m c d} A^{*}$, and $L_{2}^{M} \triangleright_{m c d} L_{2}^{*}$.

(2.2.1) $\left(\lambda P . Q^{M} \mid A^{M}\right) L_{2}^{M} \triangleright_{1 \delta}\left(\lambda P . Q^{M}\right) L_{2}^{M}$.

Then $\left(\lambda P . Q^{M}\right) L_{2}^{M}$ is a $\beta$-redex. Since $L_{2}^{M} \triangleright_{m c d} L_{2}^{*}$, by Lemma 3.2.11, there exists a term $L_{2}^{0}$ such that $L_{2}^{*} \equiv_{\mathfrak{A}} L_{2}^{0}$ and $\left(\lambda P . Q^{N}\right) L_{2}^{0}$ is a $\beta$-redex. Choose $T \equiv\left(\lambda P . Q^{*}\right) L_{2}^{0}$.

(2.2.2) $\left(\lambda P . Q^{M} \mid A^{M}\right) L_{2}^{M} \triangleright_{1 \delta} A^{M} L_{2}^{M}$.

By contradiction we can show that $\left(\lambda P . Q^{*} \mid A^{*}\right) L_{2}^{*} \triangleright_{1 \delta} A^{*} L_{2}^{*}$. Choose $T \equiv A^{*} L_{2}^{*}$.

\section{Theorem 4.2.2. (The Church-Rosser Theorem for $\beta \delta$-reduction)}

For any terms $L, M$, and $N$, if $L \triangleright_{\beta \delta} M$ and $L \triangleright_{\beta \delta} N$, then there exists a term $T$ such that $M \triangleright_{\beta \delta} T$ and $N \triangleright_{\beta \delta} T$.

Proof. Using the fact that our new $\triangleright_{\beta \delta}$ allows $\equiv_{\mathfrak{A}}$, and a single $\equiv_{\mathfrak{A}}$ is an $\triangleright_{\text {mcd }}$, the proof remains the same as the original. (Vejjajiva, 1997, pp. 64-65)

Since the congruence $\equiv_{\mathfrak{A}}$ is encapsulated within the reduction $\triangleright_{\beta \delta}$, we can define $\beta \delta$-normal forms and $\beta \delta$-equality in exactly the same way as in the original lambda calculus with patterns. (Vejjajiva, 1997; Vejjajiva \& Hall, 2002) By using the fact that the new $\triangleright_{\beta \delta}$ allows $\equiv_{\mathfrak{A}}$, and a single $\equiv_{\mathfrak{A}}$ is an $\triangleright_{m c d}$, the standard results remain true and the proofs remain unchanged except for some modifications to include the new congruence $\equiv_{\mathfrak{A}}$.

\section{Conclusion}

We have extended the concept of computability to functions on an arbitrary first-order structure using the lambda calculus with patterns. In doing so, we added a new congruence, congruence in a structure, which we proved to preserve all the basic properties of the original lambda calculus including the Church-Rosser Theorem. It is interesting to notice that, 
when defining patterns using the non-logical symbols from a language, only the function symbols which represent oneto-one functions are allowed in a pattern. Such a constraint is neccessary for the validity of the Church-Rosser theorem. For example, if we are allowed to use the symbol $\mathbf{A}$, which represents the addition function on the natural numbers, in patterns, then $(\lambda \mathbf{A} x y \cdot x) \overline{2} \equiv_{\mathfrak{A}}(\lambda \mathbf{A} x y \cdot x)(\mathbf{A} \overline{1} \overline{1}) \triangleright_{\beta} \overline{1}$ and $(\lambda \mathbf{A} x y \cdot x) \overline{2} \equiv_{\mathfrak{A}}(\lambda \mathbf{A} x y \cdot x)(\mathbf{A} \overline{0} \overline{2}) \triangleright_{\beta} \overline{0}$, but $\overline{1}$ and $\overline{0}$ do not reduce to anything common, so the Church-Rosser theorem would fail to hold.

The remaining task is to justify the word "computable". By Church's Thesis, it is widely accepted that a function on $\mathbb{N}$ is computable if and only if it is recursive (Kechris, p.3.30). For further information about recursive functions and computability see Rogers, 1992. If it could be shown that a function on $\mathbb{N}$ is recursive if and only if it is computable relative to $\mathfrak{N}$, the standard structure for the natural numbers, then we would have strong evidence to justify the use of the word "computable". The forward direction of such a proof should be straightforward, involving only the construction of terms representing the initial functions, composition, primitive recursion, and the restricted $\mu$-operator. A proof of the converse appears more challenging. It would most likely be done via arithmetization (Mendelson, 1997), whereby each term in the lambda calculus with patterns is encoded by a Gödel code and the reduction of an encoded term is done using recursive functions. The authors are currently working on this approach.

\section{References}

Barendregt, H.P. (1997). The Impact of the Lambda Calculus in Logic and Computer Science. The Bulletin of Symbolic Logic, 3 (2), 181-215.

Barendregt, H.P. (2001). The lambda calculus: Its syntax and semantics (5th impression). Amsterdam: Elsevier Science B.V.

Blum, L., Shub, M., \& Smale, S. (1989). On a theory of computation and complexity over the real numbers: $N P$ Completeness, recursive functions and universal machines. Bullentin of the American Mathematical Society, 21 (1), 1-46.

Cardone, F., \& Hindley, J. R. (2006). History of Lambda-calculus and Combinatory Logic. Handbook of the History of Logic: Vol. 5. Amsterdam: Elsevier Science B.V.

Hindley, J. R., \& Seldin, J. P. (1986). Introduction to combinators and $\lambda$-calculus. Cambridge: Cambridge University Press.

Kechris, A. S. Mathematical logic and axiomatic set theory. (Lecture Notes).

Koepke, P. (2005). Turing Computations on Ordinals. The Bulletin of Symbolic Logic, 11 (3), 377-397.

Mendelson, E. (1997). Introduction to mathematical logic. Florida: Chapman \& Hall/CRC.

Rogers, H. (1992). Theory of recursive functions and effective computability (3rd printing). Cambridge: MIT Press.

Santos, E.S. (1971). Computability by Probabilistic Turing Machines. Transactions of the American Mathematical Society, 159, 165-184.

Vejjajiva, P. (1997). A Lambda-Calculus with Patterns. Master's Thesis. Department of Mathematics, Chulalongkorn University.

Vejjajiva, P. \& Hall, M. E. (2002). A Lambda-Calculus with Patterns. Proceedings of the International Conference on Algebra and Its Applications (ICAA 2002), 266-277. 